\title{
"Nationale" Geschichte
}

Im Nachhinein besehen hat es seine Berechtigung, eine Erzählung von Südsudans "nationaler« Geschichte mit der Übergangsperiode nach der Unterzeichnung des CPA zu beginnen. Historisch-institutionell liegt der Bruch mit dem sudanesischen System in der Einrichtung einer südsudanesischen Regierung, dem Government of South(ern) Sudan (GoSS), dessen Name nach der Unabhängigkeit beibehalten wird, und einer graduellen Ablösung von den gesamtsudanesischen Strukturen. Doch darf nicht vergessen werden, dass dieser Blick durch den späteren Ausgang der Geschichte geprägt ist. Unmittelbar nach dem CPA gilt der Ausgang des Referendums keineswegs als gesichert. Beiderseitige politische Initiativen, unter wechselnder internationaler Unterstützung, versuchen, die Stimmung in die eine oder andere Richtung $\mathrm{zu}$ beeinflussen. Konzepte wie Föderalismus und Konföderalismus werden wiederholt in die politische Debatte geworfen, jedoch ohne jemals Verbindlichkeit erlangen zu können.

Die in dieser Phase in der SPLM/A fast vollständig versammelte Elite des Südsudan verhält sich, wie es ein Blick auf die vorangegangene Geschichte vermuten lässt: ambivalent. Während sich internationale Partnerstaaten und -organisationen ebenso wie zahlreiche Beobachter:innen in der Frage ergehen, ob und wie ein möglicher neuer Staat überlebensfähig sein und wie eine vollständige Trennung mit dem Norden praktisch vonstattengehen könnte, konzentriert sich die SPLM/A auf die Bearbeitung ihrer inneren Spannungslinien. Sie vermengt geschickt die Inklusion abgespaltener Milizen mit der Ausgabe gemischter Botschaften. Während sich die National Convention der SPLM im Mai 2008 zur »New Sudan«-Vision in einem vereinigten Sudan bekennt, werden anderslautende Stellungnahmen vernehmbarer. Auch von Salva Kiir selbst, der im Oktober 2009 deutliche Worte spricht: 
»When you reach your ballot boxes the choice is yours: you want to vote for unity so that you become a second class in your own country, that is your choice. If you want to vote for independence so that you are a free person in your independent state, that will be your own choice and we will respect the choice of the people. ${ }^{1}$

Eine offene Deklarierung für die Unabhängigkeit seitens der SPLM/A erfolgt aber erst einen Monat vor der Abstimmung, als die Stimmungslage bereits eindeutig ist. ${ }^{2}$ Dieses so spät geäußerte Bekenntnis kann als Hinweis dafür gesehen werden, dass der Ausgang der Transitionsperiode nach Abschluss des CPA politisch tatsächlich um einiges offener war, als es im Nachhinein den Anschein hat. Diese Periode repräsentiert eine sich aus der langen Phase des sich vereinheitlichenden südsudanesischen Widerstandes ergebende critical juncture. Sie ist ein Kulminationspunkt, der die institutionelle Konfiguration für die folgende historische Periode bestimmen wird.

»Though institutional drift [im gegenständlichen Fall die langfristige Institutionalisierung eines südsudanesischen Widerstandes über das vorangegangene Jahrhundert] leads to small differences, its interplay with critical junctures leads to institutional divergence, and thus this divergence then creates the now more major institutional differences that the next critical juncture will affect.« (Acemoglu und Robinson, 2012: 432)

Dass sich diese institutionelle Neukonfiguration in einer segmentierten und gewaltsamen Form vollzieht, ist ein schwerer und, den in solchen Prozessen steckenden Zufall bewusst mit reflektierend, unglücklicher historischer Ballast. Mit diesem Ballast startet der Südsudan das Projekt seiner Nationalstaatlichkeit.

\section{Unabhängigkeit}

Die Transitionsphase beginnt mit einer Erschütterung. Frisch als neuer Erster Vizepräsident des Sudan angelobt, befindet sich John Garang am Abend

1 Reuters, 31. Oktober 2009. „S. Sudan president makes first call for independence«, https://www.reuters.com/article/us-sudan-south-independence-idUSTRE59U1 FT20091031 [16-01-2021]. BBC, 11. Dezember 2010, „Sudan's former rebel SPLM backs independence for south «, https://www.bbc.com/news/world-africa-11976784 [16-01-2021]. 
des 30. Juli 2005 an Bord des ugandischen Präsidentenhelikopters am Rückflug von einem Treffen mit Ugandas Präsidenten Yoweri Museveni auf dessen Landsitz in Rwakitura. Wahrscheinlich war sein Ziel ein kleiner, von ihm gegründeter Ort namens New Cush nahe der kenianischen Grenze. Der Helikopter verschwindet. Erst auf mehrfache Nachfrage Ugandas nach dessen Verbleib beginnt die Suche. Am 1. August wird der Absturz des Helikopters im bergigen Dschungelgebiet Eastern Equatorias und der Tod John Garangs bekannt gegeben. Die Folgen sind sofort spürbar.

»The violence and widespread grief surrounding Garang's death forced most in the capital to lock themselves inside their homes while shop owners shuttered their stores. >Murderers! Murderers! yelled some southern Sudanese protesters who alleged the Sudanese government, which had battled Garang's rebel force for two decades before this year's peace deals, may have been behind the crash «. ${ }^{3}$

Der Nachfolgekampf innerhalb der SPLM/A beginnt unmittelbar, wird aber angesichts der Krisensituation schnell entschieden. In seinem ersten Treffen nach Garangs Tod ernennt das SPLM Leadership Council Salva Kiir zu seinem Nachfolger. Diese Wahl ist zugleich überraschend wie erwartbar. Kiir war noch wenige Jahre zuvor von Garang aus den CPA-Verhandlungsrunden abgezogen worden und verkörperte, deutlich wie nur wenige in der obersten SPLM/A-Führung, das Bekenntnis zur südsudanesischen Eigenstaatlichkeit. Damit repräsentiert er einen gewissen Bruch mit Garang. Zugleich ist er aber, als einer der letzten ihrer noch lebenden Gründungsmitglieder, insbesondere in der SPLA exzellent vernetzt und kann auf zahlreiche loyale Kommandanten zählen. Seine militärischen Fähigkeiten sind ebenfalls weitgehend unbestritten. Wenngleich weder mitreißender Redner noch überzeugender Politiker, sind seine persönlichen Fähigkeiten für die auch innerorganisatorisch fragile Übergangsphase von strategischer Bedeutung: »he is a conciliatory and unifying leader, has a gift for reading people, solid political judgment, and an ability to balance competing interests and tribal loyalties. (Natsios, 2012: 178).

Und wirklich gelingt es Kiir, nach nur zwei Wochen als Präsident des GoSS nun als Erster Vizepräsident des Sudan vereidigt, im Verein mit Garangs Witwe Rebecca Nyandeng de Mabior die wütenden Massen zu 
beruhigen und die grassierenden Verschwörungstheorien um Garangs Tod weitgehend zum Verstummen $\mathrm{zu}$ bringen (Fegley, 2011: 237). Kiirs erster politischer Schritt signalisiert politischen Ausgleich. Er ernennt Riek Machar zum GoSS-Vizepräsidenten. Ebenso bringt er andere SPLM/A-Abtrünnige wie Paulino Matip zurück in die Organisation. Andere Konkurrenz wird bewaffnet bekämpft. So wird etwa die Nuer White Army, eine starke und in ihrer Tradition, wenn nicht immer unter diesem Namen, weit zurückreichende Nuer-Miliz in Jonglei und Upper Nile (Young, 2007: 11), ab Dezember 2005 in zum Teil blutigen Kämpfen entwaffnet (Arnold und Alden, 2007). Diese gewaltsame Entwaffnungskampagne erweist sich kurzfristig als wirksam, langfristig aber, aufgrund der dadurch angeheizten ethnopolitischen Spannungen zwischen Dinka und Nuer, als desaströs.

Dennoch zeigt Kiirs innerparteiliche Versöhnungs- und Vereinheitlichungsinitiative generell positive Auswirkungen auf die Einheitlichkeit der SPLM/A. Diese Einheitlichkeit ist für die Entwicklung der Interimsphase und die sich darin entwickelnden Spannungen von entscheidender Bedeutung.

Brot und Butter des CPA-Kompromisses auch innerhalb der SPLM/A ist die Aufteilung der Öleinkommen, die diese innere Konsolidierung ermöglichen soll. Massive Aufwendungen für die Streitkräfte sind notwendig. Schon im Jahr 2009 betragen die Ausgaben laut dem damaligen US-Sondergesandten für den Sudan, Andrew Natsios (2012: 207), über eine Milliarde US-Dollar. $89 \%$ dieser Summe müssen für den Sold der SPLASoldat:innen aufgewendet werden, für 125.000 Männer und Frauen ihrer regulären Streitkraft, sowie für 175.000 weitere, die im Zuge der erwähnten Konsolidierungsbemühungen aus den verschiedenen Milizen in die SPLA integriert werden. Bei diesen irregulären Kräften handelt es sich zu einem nicht unwesentlichen Anteil um zuvor von Khartum finanzierte Gruppen, die mit der SPLA bewaffnete Auseinandersetzungen austrugen. Deren Bezahlung wird also zu einer unmittelbaren Frage der Gewährleistung von Sicherheit.

Vor dem Hintergrund dieser Kosten kommt der zukünftigen geografischen Verortung der Ölfelder, die auf Sicht die einzige verlässliche substanzielle Einnahmequelle des Südsudan darstellen, eine existenzielle Bedeutung zu. Damit wird eine von Nord und Süd anerkannte Bestimmung und Markierung der Grenzlinien entscheidend. Laut CPA sollen diese Grenzen nach dem Stand zum Zeitpunkt der sudanesischen Unabhängigkeit im Januar 1956 gezogen werden. Doch diese so einfach klingende Lösung hat zwei gewichtige Nachteile: Erstens gibt es keine unumstrittene Demarkationslinie, sondern 
eine Reihe unterschiedlich gezeichneter Karten, die um den Stichtag herum produziert wurden. Und zweitens sind die Ölfelder exakt entlang dieser unbestimmten Grenzlinie loziert, was die Verhandlungen über die nicht klar zuordenbaren Bereiche massiv erschwert. Zwar wird - letzten Endes erfolglos - versucht, die umstrittene Region Abyei durch die Einrichtung einer eigenen Abyei Border Commission ( $\mathrm{ABC}$ ) aus diesem komplizierten Demarkationsprozess herauszuhalten. Dennoch bleiben fünf zum Teil lange Grenzstreifen umstritten.

Mitunter münden die Spannungen in bewaffneten Auseinandersetzungen. In einzelnen JIUs, insbesondere in grenznahen Bereichen, kommt es zu Gefechten - Kämpfe innerhalb einer JIU in Malakal im Jahr 2007 fordern über hundert Opfer. In Abyei kommt es ab $2007 \mathrm{zu}$ bewaffneten Auseinandersetzungen mit zunehmend von den SAF unterstützten Verbänden der traditionell vom Norden her in die Region migrierenden Misseriya. In dieser Konstellation entschliesst sich die SPLM/A zu einer Verschärfung des ohnehin nur taktischen Verhältnisses mit der NCP. Im Oktober 2007 zieht sie sich aus der gemeinsamen Übergangsregierung zurück. Zudem beginnt sie, aktiv in den Konflikt in Darfur zu intervenieren. Als offensivsten Schritt lädt sie die gegen das NCP-Regime kämpfenden Rebellenverbände zu Einigungsgesprächen nach Juba. Auch wenn sich die SPLM/A bereits Ende des Jahres wieder zu einer Rückkehr in die Einheitsregierung entscheidet, zeigt sich die zunehmende Fragilität im Verhältnis der beiden CPA-Partner.

In dieser angespannten Situation hält die SPLM/A im Mai 2008 in Juba ihre zweite »National Convention « ab. Abgesehen vom symbolischen Gehalt ihrer Abhaltung führt die Konferenz zu wenig konkreten Resultaten. Angesichts der nach wie vor prävalenten innerorganisatorischen Spannungen ist dies jedoch kein schlechter Ausgang. Noch wenige Tage vor den Wahlen zum Führungsgremium deklarieren zahlreiche Parteigranden Kampfkandidaturen um verschiedene Ämter. Riek Machar plant etwa, sich gegen Salva Kiir um den Vorsitz zu bewerben. Kiir wiederum ist kontroversen Schritten nicht abgeneigt. Er portiert Versuche, Machar im Führungsgremium durch den Garang-Loyalisten James Wani Igga zu ersetzen und den SPLM-Generalsekretär Pagan Amum durch Taban Deng Gai abzulösen.

Diese Wahlvorschläge setzen die Delegierten unter den spürbaren Druck eines veritablen Sicherheitsdilemmas, zwischen parteiinterne Fronten zu geraten (Akech Thiong, 2018: 625). Letztlich gelingt es doch, durch einen Kompromiss Einheit zu bewahren, wobei die Vorbereitung auf die im Folgejahr angesetzten Wahlen als Katalysator dienen (Moro et al., 2017: 20). Schließlich 
endet die Convention versöhnlich: Kiir wird einstimmig zum Vorsitzenden gewählt und Riek Machar wird als Nummer Zwei der Organisation bestätigt. James Wani Igga, dessen Nominierung im Vorfeld auf massive Ablehnung gestoßen war, sowie Malik Aggar als Kompromisskandidat werden als Kiirs Stellvertreter eingesetzt.

Programmatisch einigt sich die SPLM in ihrer Versammlung auf ein neues Manifest. Dieses Manifest beinhaltet die bislang klarste Artikulation der »New Sudan«-Vision, wobei sie versucht, sich klar gegenüber einem auf Rassismus und Ausbeutung errichteten »alten « Sudan abzugrenzen (SPLM, 2008: 14-16). Es sind diese Formulierungen, die viele Kommentator:innen dazu verführen, die im Manifest von 2008 so deutlich erscheinenden Worte auf die gesamte SPLM/A-Geschichte davor und danach zu projizieren. Das ist jedoch ein Missverständnis. Selbst im Manifest ist die strategische Ambivalenz in der Darlegung an einigen Stellen abzulesen. Sollte sich die »New Sudan«-Vision als nicht umsetzbar erweisen, wohlgemerkt innerhalb der verbleibenden drei Jahre bis zum angesetzten Unabhängigkeitsreferendum, bliebe nur eine Wahl: »the country breaks up« (ebd.: 14).

Im Zuge der Vorbereitung auf die ursprünglich für Mitte des Jahres 2009 geplanten und dann im April 2010 abgehaltenen Wahlen bricht der nur mühsam gekittete Zielkonflikt wieder auf. Zunächst wird der aus dem »neuen Süden« des nördlichen Sudan stammende Yasir Arman als Präsidentschaftskandidat gegen al-Bashir aufgeboten. Früh zeichnet sich jedoch eine Boykottbewegung nordsudanesischer Kräfte $a b$. Insbesondere die bewaffneten Gruppen in Darfur verweigern eine Teilnahme an den Wahlen. Das heizt die Diskussionen innerhalb der SPLM/A an. Letztlich kann sich Kiir gegen die die Wahlteilnahme befürwortenden, der »New Sudan«-Orientierung zugewandten Gruppen vor allem in Khartum domizilierter Kader durchsetzen. Die SPLM/A entscheidet sich für einen Boykott der nationalen - nicht der gleichzeitig stattfindenden südsudanesischen - Wahlen. Yasir Arman verbleibt aus rechtlichen Gründen allerdings auf dem Stimmzettel.

National fällt das Resultat für die regierende NCP nicht nach Wunsch aus. Omar al-Bashir gewinnt zwar, erreicht aber nur $68 \%$ der Stimmen, während der gar nicht antretende Yasir Arman nahezu $22 \%$ der Stimmen erhält. Zum Vergleich: der zweimalige frühere Premier Sadiq al-Mahdi bleibt unter einem Prozent der abgegebenen Stimmen. Im Süden gewinnt Salva Kiir mit $93 \%$ der Stimmen klar gegen Lam Akol, der sich zuvor unter dem Namen SPLM-Democratic Change abgespalten hatte, um eigenständig kandidieren $\mathrm{zu}$ können. Auch in den Parlamentswahlen schwingt die SPLM nahezu konkur- 
renzlos oben aus. Das unvorhersehbare und spannungsgeladene Element der Wahlen im Süden liegt jedoch auf Ebene der zehn Gliedstaaten, vor allem in der Wahl der Gouverneure. Hier kommt es in einigen Teilen tatsächlich zu gröberen Schwierigkeiten. Insbesondere in Unity State und in Jonglei entladen sich die schon länger aufgestauten lokalen wie nationalen politischen Konflikte in einer Weise, die den kommenden südsudanesischen Bürgerkrieg erahnen lässt.

Bei der Wahl zum Gouverneur von Unity State schlägt der vom SPLM/AEstablishment favorisierte und formell von der Partei nominierte Taban Deng die ebenfalls kandidierende und in den Nuer-Gemeinschaften überaus beliebte Angelina Jany Teny, die Ehefrau von Riek Machar. Teny ist als unabhängige Kandidatin von Beginn an mit heftigem Gegenwind konfrontiert. Ihr Wahlkampfbüro wird geschlossen, Unterstützer:innen von ihr werden bedroht und zum Teil verhaftet. Fälschungen sind nicht nur wahrscheinlich, sondern wahrscheinlich massiv. Medien berichten nach Auszählungen von acht der neun Counties anfänglich von einem klaren Vorsprung Tenys, was auf politischen Druck hin revidiert werden muss. Das Endergebnis weist Taban mit mehr als doppelt so vielen Stimmen als Teny aus (137.662 gegenüber 63.651) ${ }^{4}$. Nach der Bekanntgabe des Resultates kommt es zu Unruhen in Bentiu, im Zuge derer zwei Teny-Sympathisanten erschossen werden.

Taban Deng, dem schon zuvor ein allzu offenkundiger Griff nach den Öleinnahmen nachgesagt wird, setzt sich schlussendlich aber durch, um den Preis des Verlustes jeglichen Rückhalts in den Nuer-Gemeinschaften. Diese prekäre Situation zwingt ihn, die aktive Aussöhnung mit Machar zu suchen, was wiederum sein Verhältnis zu Kiir belastet. Schließlich wird Taban Deng im Juni 2013 per präsidentiellem Dekret ohne weitere Begründung aus seiner Position entlassen. Kiir setzt den vorangegangenen Gouverneur von Unity und langjährigen persönlichen Kontrahent von Taban Deng, Joseph Nguen Monytuil, als dessen Nachfolger ein. ${ }^{5}$ Diese Entlassung, offenbar Teil einer weiterreichenden gezielten Entmachtung von Machar-Loyalist:innen, lässt sich bereits als Vorbote eines heraufziehenden Bürgerkrieges interpretieren. [12-01-2021]. 
In Jonglei wiederum rebelliert der ehemalige stellvertretende Generalstabschef der SPLA, George Athor Deng, mit der von ihm neu formierten Miliz South Sudan Democratic Movement/Army (SSDM/A), nachdem er die Gouverneurswahl gegen den Amtsinhaber Kuol Manyang Juuk verliert (Thomas, 2015: 215-216). Die SSDM/A kann viele gegen die SPLA eingestellte Murle mobilisieren. Letztlich führt die Rebellion zu einigen erfolglosen Waffenstillständen, schwelt aber trotz des Todes von Athor im Dezember 2011 noch bis Mitte des Jahres 2013 weiter.

Unmittelbar nach den Wahlen starten die Vorbereitungen für das für Januar 2011 angesetzte Unabhängigkeitsreferendum. Die Stimmberechtigung beruht ebenfalls auf dem umstrittenen gesamtsudanesischen Zensus von 2008 , dem bis heute letzten, der im Südsudan durchgeführt wurde. Angesichts der großen Zahl von Südsudanes:innen, die in den vergangenen Jahrzehnten vor dem Krieg aus der Region geflüchtet waren, ist es wenig überraschend, dass Fragen der Stimmberechtigung und, damit zusammenhängend, der Staatsbürgerschaft höchst umstritten sind. Referendumsgesetz und Referendumskommission, beides unerlässliche Vorbedingungen für die Durchführung, werden erst verspätet beschlossen und eingesetzt, was den Prozess zusätzlich verkompliziert (Marzatico, 2011).

Schließlich wird die Wahlberechtigung vom Referendumsgesetz in einer Weise festgeschrieben, die bis heute die Anspruchsberechtigung für die südsudanesische Staatsbürgerschaft definiert. Stimmberechtigt, und damit als Südsudanes:innen definiert, sind demnach Personen, von denen entweder zumindest ein Elternteil eine Zugehörigkeit zu den »indigenous communities residing in Southern Sudan« mit dem Stichtag 1.1.1956 aufweist oder die eine länger zurückreichende Abstammung »to one of the ethnic communities« nachweisen können. Ebenso zugelassen sind Personen, die selbst oder deren Eltern permanent und ohne Unterbrechung im südlichen Sudan leben. Dieser »ethnic turn« zur Abstammungslogik (jus sanguinis, vgl. Bakhit, 2016) führt zu einer spezifischen Ökonomie, die für jene, die in der Lage sind, glaubwürdige Zertifikate auszustellen oder darüber Entscheidungen zu treffen, finanziell überaus einträglich ist (Marko, 2015).

Je näher das Referendum rückt, desto mehr laden sich die Spannungen auf. Sowohl SPLA als auch SAF konzentrieren kampfbereite Truppen in Grenznähe. Die gewaltsamen Zusammenstöße beschränken sich allerdings auf das umstrittene Abyei, dort jedoch mit umso signifikanteren Auswirkungen. Die Frage von Abyeis Grenzen war schon zuvor der internationalen Arbitration übergeben worden. Die in Den Haag im Juli 2009 getroffene 
Entscheidung fällt tendenziell zugunsten Khartums aus: das ölreiche traditionelle Dinka-Siedlungsgebiet von Panthau (in Dinka) oder Heglig (in Arabisch), wird entgegen südsudanesischer Ansprüche als außerhalb von Abyei gelegen beurteilt (wenngleich sich die spezifische Frage einer Inklusion dieses Ortes eigentlich weder in der Arbitration noch in den Feststellungen der $A B C$ nie stellte und erst zu einem späteren Zeitpunkt relevant wurde, vgl. Johnson, 2012).

Dennoch will es Khartum nicht auf ein Referendum über Abyeis Zugehörigkeit, das ja laut CPA am selben Tag wie das Unabhängigkeitsreferendum stattzufinden hatte, ankommen lassen. Auch in Abyei geht es, nochmals zugespitzt, um die Frage der Wahlberechtigung. Die dem Norden zugerechneten, saisonal nach Abyei migrierenden Misseriya unterhalten keine permanenten Niederlassungen in der Region, was die ansässigen Ngok Dinka, die in relevanter Zahl in höheren Chargen der SPLA vertreten sind, dazu veranlasst, ihnen das Wahlrecht streitig zu machen. Noch vor dem Referendum brechen Kämpfe aus, wobei Misseriya-Verbände durch SAF-Einheiten unterstützt werden. Offenbar geht es um einen strategischen Versuch, das AbyeiReferendum zu unterbinden (Johnson, 2016a: 173). Dies gelingt. Abyei bleibt bis zum Abschluss eines Interimsvertrages ${ }^{6}$ zwischen den beiden Seiten im Juni 2011, der die Einrichtung einer UN/AU-Friedensmission (UNISFA, bestehend aus äthiopischen Truppen) mit einschließt, faktisch durch die SAF okkupiert (Rolandsen und Daly, 2016: 147).

Das Unabhängigkeitsreferendum findet jedoch, wider alle negativen Umstände, unter Beobachtung von Carter Center, Europäischer Union, Afrikanischer Union, der Arabischen Liga und IGAD, in der zweiten Januar-Woche des Jahres 2011 statt. Das Ergebnis ist eindeutig. Nur 44.888 Stimmen werden für den Verbleib in einem gemeinsamen Staat abgegeben, 3.8 Millionen, fast $99 \%$, stimmen für die Unabhängigkeit. Die Reaktion des Nordens ist überraschend. Bashir akzeptiert das Ergebnis bereits, bevor das offizielle Endresultat vorliegt, und gratuliert zur Unabhängigkeit. Nach einer kurzen Namenssuche - historische Reminiszenzen an die Anya-nya-Zeit wie Nile Republic oder Azania werden geprüft - fällt die Entscheidung auf South Sudan.

Unmittelbar nach dem Referendum setzt Salva Kiir per präsidentiellem Dekret ein Constitutional Drafting Committee ein, das von SPLM/A-Kadern do- 
miniert wird. In einem exklusiven Prozess wird ein schneller Entwurf einer Übergangsverfassung gebastelt (LeRiche und Arnold, 2013: 152-153), der auf der im Jahr 2005 eingeführten Übergangsverfassung für den südlichen Sudan beruht. Diese war selbst wiederum in den meisten Aspekten - wie die Rechte und Prozesse von Exekutive und Legislative - eine Kopie der sudanesische Übergangsverfassung von 2005. Parteiintern kommt es zu zahlreichen Einwänden gegen diesen Prozess. Riek Machar will die präsidentielle Machtfülle reduzieren. Diesen Einwürfen wird nicht stattgegeben, mit dem Argument, es gehe ohnehin nur um ein Interimsdokument, das nach den für 2015 angesetzten Wahlen neu geschrieben werden müsste.

Im Rahmen einer opulenten Feier vor hunderttausenden Gästen, unter ihnen Omar al-Bashir, am Platz des Garang-Memorials in Juba wird am 9. Juli 2011 formal die Unabhängigkeit ausgerufen. Für einen Moment sind alle Querelen vergessen. In weiten Teilen des Landes ist eine Aufbruchsstimmung spürbar. Mit internationalen Hilfsgeldern wird eine eigene Währung installiert, eine Woche nach der Unabhängigkeit ersetzt das Südsudanesische Pfund (SSP) das sudanesische, zunächst, wenn auch nur für kurze Zeit, zu einem Wechselkurs von $1: 1$.

In den ersten Monaten nach der Unabhängigkeit setzt Kiir seine Politik der Wiedereingliederung abtrünniger Kommandeure und konkurrenzierender Milizen fort. In Unity State wird ein Waffenstillstand mit dem seit den Gouverneurswahlen rebellierenden Gatluak Gai ausgehandelt. Gatluak Gai überlebt dieses Abkommen jedoch gerade einmal um vier Tage, bevor er von einem mit Khartum kollaborierenden Milizionär erschossen wird. In Jonglei kommt es im August $2011 \mathrm{zu}$ heftigen Kämpfen mit mehreren hundert Toten. Doch auch dort schließt Kiir Frieden. Nuer-General Peter Gadet und Murle-Rebellenführer David Yau Yau werden in die SPLA eingegliedert. Weniger Glück hat der ebenfalls seit den Wahlen von 2010 aufständische George Athor. Er zeigt sich nicht zu einem Abkommen bereit und wird im Dezember 2011, wahrscheinlich von südsudanesischen Grenzschutzeinheiten an der Grenze zum Sudan, erschossen.

Dieser von Kiir seit dem Abschluss des CPA verfolgte Ansatz erhält bald den Namen »big tent«. Ein solches großes Zelt soll gewährleisten, dass nach Prinzipien des individuellen wie kollektiven politischen und ökonomischen Gewinns am mehrschichtigen südsudanesischen politischen Marktplatz eine möglichst breite Koalition um Kiir gebildet wird, die nachhaltig aufrechterhalten werden kann. Es ist unklar, woher die Bezeichnung »big tent« konkret stammt. Vermutlich handelt es sich um eine analytische Zuschreibung, 
die auf den politikwissenschaftlichen Begriff einer »big tent«-Koalition (vgl. Weinstein und Goldstein, 2012) zurückgeht. Erste Erwähnungen im südsudanesischen Kontext finden sich in Analysen der International Crisis Group (zunächst als »large tent«, ICG, 2011: 13, später als »big tent«, ICG: 2014: 5; LeRiche und Arnold, 2013: 199 verweisen auf die umgangssprachliche Verwendung des Ausdrucks im südsudanesischen politischen Diskurs jener Zeit).

Auf politisch-strategischer Ebene lässt sich »big tent« auf die Juba Declaration $^{7}$ zurückführen, die im Januar 2006 mit der von Paulino Matip kommandierten, in Unity State einflussreichen Nuer-Miliz South Sudan Defence Forces (SSDF) unterzeichnet wurde. Schon im Untertitel führt die Deklaration die Begriffe »Unity« und »Integration«. Sie zeichnet damit den konkreten Umsetzungspfad des »big tent « vor: Rebellion wird mit einer Generalamnestie und der Reintegration des Führungspersonals in verantwortlichen Positionen der SPLA befriedet. Diese Vorgangsweise verankert im ohnehin zur Gewalt tendierenden südsudanesischen politischen Marktplatz ein unglückliches Anreizsystem. Paulino Matip erlangt auf diesem Weg die Position als Stellvertretender Oberkommandierender der SPLA.

Abgesehen von den problematischen Anreizen ist der »big tent«-Ansatz kostspielig. Im Jahr 2012 zählt die SPLA 150.000 aktive Soldat:innen und mehrere hundert Generäle. Deren Saläre sind, im Vergleich mit zivilen Beschäftigungen oder dem Sold der Armeen in den Nachbarstaaten, hoch. In den höheren Rängen ist oftmals eine gehörige Menge Geld im Spiel, die in so manchen Fällen in opulente Eigenheime in Nairobi, Khartum oder Kampala investiert werden. Die finanzielle Unterfütterung dieser spezifischen politischen Marktökonomie liefern die Gewinne aus der Ölförderung. Von daher überrascht es wenig, dass es dieser Sektor ist, der bald nach der Unabhängigkeit aufgrund bestehender Uneinigkeiten über die Pipeline-Gebühren erste schwerwiegenden Auseinandersetzungen mit dem neuen Nachbarn im Norden provoziert. »The North borrowed billions to finance this oil infrastructure, and it wanted the South to assume a portion of this debt." (Natsios, 2012: 211)

Zwar kommt es im September 2011 mit substanzieller internationaler Unterstützung zu einer vorübergehenden vertraglichen Übereinkunft, jedoch gerät der Südsudan aufgrund vorheriger Unterbrüche in der Ölproduktion unmittelbar in Zahlungsschwierigkeiten. Im Dezember beginnt Khartum, südsudanesisches Öl auf eigene Faust abzuzweigen, um Zahlungen zu er- 
zwingen. Juba beantwortet dies mit der Einstellung der Ölförderung im Januar 2012 (de Waal, 2014).

Die Spannungen eskalieren im darauffolgenden April, als die SPLA das im Sudan gelegene, aber beidseitig beanspruchte Panthau/Heglig (vgl. Johnson, 2012), in dem sich ertragreiche Förderanlagen befinden, militärisch attackiert und einnimmt. Trotz eines Rückzuges nach zehn Tagen, der je nach Sichtweise wegen eines erfolgreichen Gegenangriffes oder freiwillig erfolgt, bleibt die Lage explosiv. Erst nach massivem Druck von AU, UN, und zuletzt der USA beginnen beide Seiten zu verhandeln. Sie unterzeichnen im September 2012 eine Serie von neun bilateralen Verträgen, die neben einem bedingungslosen und international überwachten Rückzug der Streitkräfte beider Seiten hinter die provisorisch festgelegten Landesgrenzen unter anderem die PipelineGebühren regeln. Diese werden gegenüber der vorher bestehenden Regelung etwas herabgesetzt. »Processing«, »transport« und »transit fee« zusammengenommen belaufen sich nun, abhängig von der sudanesischen Beteiligung am jeweiligen Konsortium, auf 9,10 oder 11 US-Dollar per Barrel. Zugleich verpflichtet sich der Südsudan zur sofortigen Begleichung von drei Milliarden US-Dollar an Außenständen. ${ }^{8}$

Diese Abkommen deeskalieren die Situation, führen jedoch $\mathrm{zu}$ keiner letztendlichen Beseitigung der bilateralen Probleme. Bereits unmittelbar nach der Unabhängigkeit hatte der ehemalige Northern Sector der SPLM/A, der nun eigenständig als SPLM-North (SPLM-N) agiert, den Kampf gegen das sudanesische Regime aufgenommen. Die SPLM-N sieht die unpräzisen Regelungen im CPA nicht erfüllt und erhebt gegenüber Khartum Forderungen in Richtung Föderalismus, Demokratisierung und Säkularisierung. Spätestens seit den Wahlen im Jahr 2010 sind politische Auseinandersetzungen an der Tagesordnung (vgl. Kunda Komey, 2013). Während das sudanesische Regime die SPLM-N im September 2012 offiziell verbietet, sucht diese wiederum den aktiven Kontakt zu darfurischen Rebellengruppen, um eine geeinte Kampffront in den peripheren Regionen des Sudan zu eröffnen, die Sudan Revolutionary Front (SRF). Trotz einer späteren Spaltung der SPLM-N im Jahr 2017, die zu internen bewaffneten Auseinandersetzungen führt, wird die

8 Agreement between The Government of the Republic of South Sudan and The Government of the Republic of the Sudan on Oil and Related Economic Matters, unterzeichnet am 27. September 2012, https://www.peaceagreements.org/viewmasterdocu ment/1076. 
SRF tatsächlich aktiv bleiben. Sie entwickelt sich zu einem der wesentlichen Akteure des Juba-Friedensprozesses für den Sudan im Jahr 2020.

\section{Der Weg in den Bürgerkrieg}

Irgendwann im Zuge des heißen Konfliktes mit dem Sudan und der wegbrechenden Öleinnahmen (eine These, die Alex de Waal, 2014, etabliert hat) entscheidet sich Salva Kiir zur Aufgabe der »big tent«-Politik. Die Auseinandersetzungen innerhalb der SPLM/A werden härter und zunehmend mit offenem Visier ausgetragen. Als auslösender Faktor erweist sich die heikle Frage einer Nachfolgeregelung für Kiir, insbesondere im Hinblick auf die für 2015 angesetzten ersten südsudanesischen Wahlen.

Der vereinbarte Ablauf sieht vor, die Wahlen für Parteivorsitz und Spitzenkandidatur im Rahmen der geplanten dritten National Convention der SPLM im Mai 2013 durchzuführen. Mitte des Jahres 2012 wirft Riek Machar als Erster seinen Hut für eine Gegenkandidatur in den Ring. Ohnehin betrachtet er Kiirs Regentschaft als eine durch den Tod Garangs notwendig gewordene Interimsperiode. Schnell folgen Rebecca Nyandeng und Pagan Amum, die ebenfalls öffentlich ihre Kandidatur deklarieren. Kiir selbst gibt sich hinsichtlich seiner Pläne bedeckt (Johnson, 2014b: 169).

Hinter den Kulissen bereiten sich alle Seiten auf eine sich zuspitzende innerparteiliche Auseinandersetzung vor. Als öffentlichkeitswirksames Instrument dient Kiir dabei eine Anti-Korruptionskampagne. Im Juni 2012 fordert er in einem offenen Brief 75 aktive oder ehemalige Staatsbedienstete auf, alle durch Korruption erschlichenen Gelder, die er auf vier Milliarden US-Dollar schätzt, zurückzuzahlen. Was zu diesem Zeitpunkt wie ein schräger PR-Stunt wirkt, zeigt im Zuge der folgenden politischen Kontroversen schnell seine eigentliche Zielrichtung. Als der Brief seine intendierte politische Druckwirkung nicht zu entfalten droht, folgen konkrete Schritte.

Genau ein Jahr nach dem Verfassen des Briefes entlässt Kiir Finanzminister Kosti Manibe Ngai, einen engen Vertrauten des SPLM-Generalsekretärs Pagan Amum, und den Kabinettsminister Deng Alor unter dem Vorwurf der Korruption.

Diese beiden Entlassungen sind der Startschuss einer sich schnell drehenden Eskalationsspirale. Diese wird noch dadurch befeuert, dass Kiir bereits Ende 2012 dazu übergegangen ist, die zwei wesentlichen Arbeitsgremien der SPLM, das Politbüro und das National Liberation Council, durch Sitzungs- 